\title{
ON GRAPHS REPRESENTING AUTOMORPHISMS OF FREE GROUPS
}

\author{
SAVA KRSTIĆ
}

(Communicated by Warren J. Wong)

For every automorphism $f$ of the free group $F=\left\langle x_{1}, \ldots, x_{m} \mid-\right\rangle$ Gersten [2, Theorem 5.6] constructed a finite graph representing $f$ and used this graph to give the first proof of Scott's conjecture that the subgroup of fixed points of $f$ is finitely generated. A simpler construction of a graph representing $f$ is contained in a more general treatment of Stallings [6]; also, as Goldstein and Turner [3] show, this graph can be obtained from Whitehead's 3-dimensional model for $f$. Apart from the applications in the study of fixed points, graphs representing automorphisms are of some independent interest; Hoare [4] uses them to reprove Whitehead's Cut Vertex lemma [7] and Thurston's Bounded Cancellation theorem [1]. This note offers another construction of Gersten's graph with a very short proof.

We say that the graph $\Gamma$ represents $f$ if $\Gamma$ has the following properties. It is finite with every edge colored blue or red and labeled $x_{i}$ or $x_{i}^{-1}(1 \leq i \leq m)$, assuming that inverse edges have the same color and inverse labels. Every $x_{i}$ is the label of at least one blue and one red edge. Equally colored edges emanating from the same vertex have different labels. The set of all blue edges, and the set of red edges as well, is the edge set of a maximal tree in $\Gamma$. Finally, there is a vertex $*$ such that for every path $p$ which begins and ends at $*$ one has blue $(p)=f(\operatorname{red}(p))$, where blue $(p)$ is the element of $F$ (a word in $x_{i}^{ \pm 1}$ ) obtained by reading out the labels of blue edges while traversing $p$, and $\operatorname{red}(p)$ is defined analogously. (This is a modified definition, an inessential variation of the original one.)

Let $T$ be the Cayley graph of $F\left[5\right.$, p. 123] and $T_{B}$ and $T_{R}$ copies of $T$ colored blue and red respectively. Let $\Gamma_{0}$ be the graph obtained by taking $T_{B}$ and $T_{R}$ together and identifying every vertex $v$ of $T_{B}$ with the vertex $f v^{-1}$ of $T_{R}$. It is readily seen that $\Gamma_{0}$ satisfies all conditions of the preceding paragraph except that of being finite.

If elements of $F$ are regarded as reduced words in $x_{i}^{ \pm 1}$, it is easy to see that a subset $A$ of $F$ containing 1 is the vertex set of a subtree of $T$ if and

Received by the editors April 25, 1988 and, in revised form, September 13, 1988.

1980 Mathematics Subject Classification (1985 Revision). Primary 20E05, 20F28. 
only if $A$ is left-closed in the sense that it contains all initial segments of its elements. Dually, right-closed subsets are subsets containing terminal segments of their elements. Finally, we say that a subset $A$ of $F$ represents $f$ if $A$ is left-closed and $f A$ is right-closed.

Now if $A$ represents $f$, then $A$ and $f A^{-1}$ are both left-closed and so determine subtrees of $T_{B}$ and $T_{R}$ respectively, which have the same vertex set in $\Gamma_{0}$. Thus $A$ determines a subgraph of $\Gamma_{0}$, which by a simple examination satisfies all conditions for being a graph representing $f$, except possibly that of being finite and that of having edges labeled $x_{i}$ for every $i$.

So we get the following algebraic restatement of the existence theorem for graphs representing automorphisms.

Theorem. If $f$ is an automorphism of $F$ then every finite subset of $F$ is contained in a finite subset of $F$ which represents $f$.

Proof. For every subset $B$ of $F$ the sets of all initial and terminal segments of elements of $B$ will be denoted respectively by $L(B)$ and $R(B)$, and $R_{k}(B)$ will stand for $R(B)$ minus all its elements of length $>k$.

Let $A_{0}$ be an arbitrary finite subset of $F$. Consider the sequence $\left(A_{n}\right)$ inductively defined by $A_{n+1}=L\left(f^{-1} R\left(f A_{n}\right)\right)$. It is increasing, and its union $A$ is a set representing $F$. Indeed, $A=L(A)$ follows from $A_{n}=L\left(A_{n}\right)$ and $f A=R(f A)$ follows from $f A_{n+1}=f L\left(f^{-1} R\left(f A_{n}\right)\right) R\left(f A_{n}\right)$. It remains to prove that $A_{n+1}=A_{n}$ for some $n$.

Lemma. If $L(B)=B$ and $k=\max \left\{\left|f x_{i}\right|: 1 \leq i \leq m\right\}$ then $L\left(f^{-1} R(f B)\right)=$ $f^{-1} R(f B) \cup L\left(f^{-1} R_{k}(f B)\right)$.

The proof of the Lemma will be given at the end.

The number of elements of length $\leq k$ in $F$ is finite, so there is $N$ such that the increasing sequence $R_{k}\left(f A_{n}\right)$ is constant for $n \geq N$. By the lemma, $A_{N+2}=f^{-1} R\left(f A_{N+1}\right) \cup L\left(f^{-1} R_{k}\left(f A_{N}\right)\right)$. Since $L\left(f^{-1} R_{k}\left(f A_{N}\right)\right) \subseteq A_{N+1} \subseteq$ $f^{-1} R\left(f A_{N+1}\right)$ it follows that $A_{N+2}=f^{-1} R\left(f A_{N+1}\right)$ and similarly that $A_{N+3}=$ $f^{-1} R\left(f A_{N+2}\right)$. So $A_{N+3}=f^{-1} R\left(R\left(f A_{N+1}\right)\right)=A_{N+2}$, finishing the proof.

Proof of lemma. We may assume $B=L(w)$ for some $w \in F$. Write $w=$ $y_{1} \cdots y_{r}$ with $y_{i} \in\left\{x_{1}, \ldots, x_{m}\right\}^{ \pm 1}$, so that $B=\left\{1, w_{1}, \ldots, w_{r}\right\}$, where $w_{i}=$ $y_{1} \cdots y_{i}$. Let $C$ be the set on the right-hand side of the equality in the lemma. We have to prove for every $u \in R\left(f w_{i}\right), 1 \leq i \leq r$, that $L\left(f^{-1} u\right) \subseteq C$. We do this by induction on $i$. The case $i=1$ follows from $R\left(f w_{1}\right)=R_{k}\left(f w_{1}\right)$.

Now suppose $u \in R\left(f w_{i}\right)$ and $|u|>k$. (There is nothing to prove if $|u| \leq k$.) Let $f w_{i}=v u$. Since $w_{i}=w_{i-1} y_{i}$ and $\left|f y_{i}\right| \leq k$, it follows that $v$ is an initial segment of $f w_{i-1}$ as well; $f w_{i-1}=v u^{\prime}$. Thus $f^{-1} u=\left(f^{-1} u^{\prime}\right) y_{i}$, and so $L\left(f^{-1} u\right) \subseteq L\left(f^{-1} u^{\prime}\right) \cup\left\{f^{-1} u\right\} \subseteq C$ because $L\left(f^{-1} u^{\prime}\right) \subseteq C$ by induction hypothesis and $f^{-1} u \in f^{-1} R\left(f w_{i}\right) \subseteq C$. 


\section{REFERENCES}

1. D. Cooper, Automorphisms of free groups have finitely generated fixed point set, J. Algebra 111 (1987), 453-456.

2. S. M. Gersten, Fixed points of automorphisms of free groups, Adv. in Math. 64 (1987), 51-85.

3. R. Z. Goldstein and E. C. Turner, Automorphisms of free groups and their fixed points, Invent. Math. 78 (1984), 1-12.

4. A. H. M. Hoare, On automorphisms of free groups I, (preprint).

5. R. C. Lyndon and P. E. Schupp, Combinatorial group theory, Springer-Verlag, Berlin, 1977.

6. J. R. Stallings, Graphical theory of automorphisms of free groups, Combinatorial Group Theory and Topology (S. M. Gersten and J. R. Stallings, eds.) Ann. of Math. Stud. no. 111, Princeton Univ. Press, Princeton, 1987, pp. 79-107.

7. J. H. C. Whitehead, On certain sets of elements in a free group, Proc. London Math. Soc. $\mathbf{4 1}$ (1936), 48-56.

Matematički Institut, 11000 Belgrade, Yugoslavia 